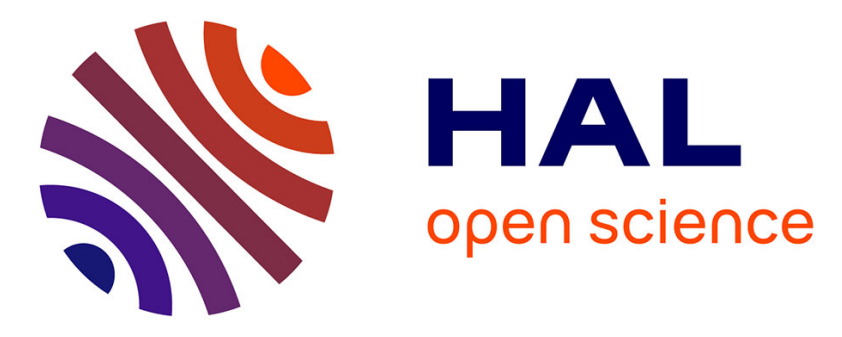

\title{
Cutaneous listeriosis, a case series of 16 consecutive patients over 25 years
}

Benoit Pilmis, Alexandre Leclercq, Mylène M Maury, Alexandra Moura, Hélène Bracq-Dieye, Pierre Thouvenot, Guillaume Vales, Marc Lecuit, Caroline Charlier, Cutaneous Listeriosis Study Group

\section{To cite this version:}

Benoit Pilmis, Alexandre Leclercq, Mylène M Maury, Alexandra Moura, Hélène Bracq-Dieye, et al.. Cutaneous listeriosis, a case series of 16 consecutive patients over 25 years. Journal of Infection, 2020, 80 (2), pp.232-254. 10.1016/j.jinf.2019.10.004 . pasteur-02586108

\section{HAL Id: pasteur-02586108 https://hal-pasteur.archives-ouvertes.fr/pasteur-02586108}

Submitted on 10 Jun 2020

HAL is a multi-disciplinary open access archive for the deposit and dissemination of scientific research documents, whether they are published or not. The documents may come from teaching and research institutions in France or abroad, or from public or private research centers.
L'archive ouverte pluridisciplinaire HAL, est destinée au dépôt et à la diffusion de documents scientifiques de niveau recherche, publiés ou non, émanant des établissements d'enseignement et de recherche français ou étrangers, des laboratoires publics ou privés. 
Cutaneous listeriosis, a case series of 16 consecutive patients over 25 years

Benoit Pilmis $^{1,2,3}$, Alexandre Leclercq ${ }^{1,2}$, Mylène M. Maury ${ }^{1,2,4}$, Alexandra Moura ${ }^{1,2,4}$, Hélène Bracq-Dieye ${ }^{2}$, Pierre Thouvenot ${ }^{2}$, Guillaume Valès ${ }^{2}$, Marc Lecuit ${ }^{1,2,3,4, *}$, Caroline Charlier $^{1,2,3,4, *}$ for the cutaneous listeriosis study group

${ }^{1}$ Institut Pasteur, French National Reference Center and WHO Collaborating Center for Listeria, ParisFrance

${ }^{2}$ Université de Paris, Necker-Enfants Malades University Hospital, Department of Infectious Diseases and Tropical Medicine, AP-HP, Paris, France

${ }^{3}$ Institut Pasteur, Biology of Infection Unit, Paris-France

${ }^{4}$ Inserm U1117, Paris-France

* Correspondence to Caroline Charlier and Marc Lecuit

Unité de Biologie des Infections

Institut Pasteur, 28 rue du Dr Roux

75015 Paris

Email: caroline.charlier@pasteur.fr and marc.lecuit@pasteur.fr

Keywords: listeriosis, skin, Listeria monocytogenes

Word count: 866 


\section{Dear Editor,}

Scobie et al. recently reported a 10 -year review of non-pregnant listeriosis cases in England ${ }^{1}$. No case of cutaneous listeriosis was reported in this large cohort of 1,357 patients. Aside gastroenteritis and invasive infections (septicemia, neurolisteriosis and maternal-neonatal infections), Listeria monocytogenes $(\mathrm{Lm})$ can also be responsible for other focal infections, including bone and joint infection, biliary, pulmonary, or urinary tract infections ${ }^{2}$. Skin infections have also been reported before, either as a part of disseminated infection in neonates — a condition known as "granulomatosis infantiseptica" - or as an occupational disease of veterinarians and farmers $^{3}$. Cutaneous listeriosis include a multistep process starting from erythematous lesions, which become nodular and pustular within 2-5 days after contact of intact skin with infected fluids, such as animal secretions ${ }^{3,4}$.

In France, surveillance of human listeriosis is based on mandatory reporting of cases since 1999 and voluntary submission of strains to the French National Reference center for Listeria (NRC). The exhaustiveness of this reporting is estimated around $87 \%^{5}$. In this context, we retrospectively studied all consecutive culture-proven Listeria-associated skin and soft tissue infections reported to the NRC from January 1994 to December 2018). All cases with the mention of "cutaneous", "nodular", "pustular", "skin" or "soft tissue” infections were included. Medical charts were reviewed and clinical bacterial isolates sequenced.

Species identification was carried out with API-Listeria microgallery (bioMérieux, Marcy l'Etoile, France) before January 2017, and thereafter with MALDI-TOF mass spectrometry as described ${ }^{6}$. Hemolysis tests were performed at $37^{\circ} \mathrm{C}$ for $24 \mathrm{~h}$ on horse blood agar. Genome sequencing was performed as described ${ }^{7}$. PCR serogrouping, multilocus sequence types (MLST) and core genome MLST (cgMLST) profiles were deduced from genome assemblies using the BIGSdb-Lm platform (https://bigsdb.pasteur.fr/listeria) ${ }^{8}$. 
Among 7,294 human cases collected between 1994 and 2018 in France, 16 involved patients with Listeria-associated skin/soft tissue infections (0.2\%). Of them, $11(11 / 16,69 \%)$ were male. Median age was 62 years (range: 8 - 93); 4 patients (4/16, 25\%) were more than 80 years old. Only 5 patients reported immunosuppressive comorbidity (lung cancer $(n=2)$, cirrhosis $(n=1)$, diabetes mellitus $(n=1)$, Rituximab therapy $(n=1))(5 / 16,31 \%)$. Lesions consisted in single skin abscesses $(n=11)$, cellulitis $(n=3)$ or single nodular lesions $(n=2)$. Three patients reported fever (3/16, 19\%). Skin lesions involved legs $(n=7 / 15,47 \%)$, arm/hands $(n=5 / 15,33 \%)$, or the face $(n=3 / 15,20 \%)$. When performed $(n=5)$, all blood cultures were negative. No patient reported any other Listeria localization, in particular no concomitant neurolisteriosis or gastro-enteritis. Environmental exposure was only reported in one farmer patient. No other patient reported any contact with animals, farms, hay or contaminated silage. No preexisting skin abrasion was reported.

$L m$ was grown from skin samples in 15 cases; Listeria seeligeri was identified in one case. One Lm isolate from 1994 could not be grown from the culture collection and was therefore not sequenced. All sequenced $L m$ isolates belonged to different cgMLST types (Figure 1), indicating that these cases were unrelated microbiologically, and that a large variety of strains can be responsible for cutaneous listeriosis. $5 / 14 \mathrm{Lm}$ isolates (36\%) belonged to hypervirulent clonal complexes $(\mathrm{CC} 1 \text { and } \mathrm{CC} 4)^{9}$. No acquired resistance toward the main antibiotics for listeriosis treatment was evidenced (amoxicillin, ampicillin, trimethoprim/sulfamethoxazole or gentamicin).

All patients received antibiotic therapy against $L m$, for a median of 12.5 days (range, $7-21$ ): amoxicillin/piperacillin 12/16, $75 \%$, cotrimoxazole in $1 / 16,6.3 \%$ and was not reported in 3 cases. Nine of them with abscesses also benefited from local drainage $(9 / 16,56 \%)$. All patients experienced full recovery. 
So far, only 26 cases of skin and soft tissue infections have been reported since 1957, as case reports or small series of less than 6 cases $^{4}$. This series of 16 cases is, to our knowledge, the largest on this rare entity. Importantly, most published cases were reported before 1995 and before the implementation of current animal and food safety regulation ${ }^{4}$. At that time, cutaneous listeriosis appeared as an occupational hazard involving veterinarians or farmers exposed to infected secretions. Our case series, starting in 1994, captured a different picture of the disease. Indeed, in sharp contrast to previous reports that involved immunocompetent patients with massive occupational exposure, only one patient reported environmental (animal) exposure, whereas all other cases had no identified source of infection, but rather reported classical risk factors for listeriosis, namely older age and immunosuppressive comorbidities ${ }^{10}$. Although no local trauma was reported, skin infections may result from direct local inoculation rather than hematogenous dissemination, considering the constant absence of concomitant bacteremia, and of any other localization of infection, as well as the favorable evolution under relatively low dose amoxicillin ( $3 \mathrm{~g} / \mathrm{d})$, much lower than recommended regimens for systemic listeriosis. The source of infection could be either contact with contaminated material, in case of infections of the limbs, or chronic fecal carriage, in case of perineal abscesses, as reported in bovine mastitis $^{11}$.

The main limitation of this work is its retrospective nature, although cases were prospectively reported as part of national surveillance. One could also not exclude under-diagnosis and underreporting of this rare presentation, as most empiric antibiotic therapy targeting skin pathogens would be effective against $L m$ and lead to complete resolution, most infections remaining therefore likely undetected.

Altogether, these results underline the wide array of infections associated with Listeria monocytogenes and the favorable outcome of this very rare clinical entity. 


\section{Figure Legend}

Figure 1. Single linkage clustering based on the cgMLST profiles of 14 Listeria monocytogenes isolates collected between 1994 and 2018 from patients' skin (1 isolate from 1994 could not be sequenced). The percentage of cgMLST profile similarities between isolates (based on 1748 genes) are indicated at branch nodes. The details on the molecular typing results, skin lesion and body location (when available) are provided in the adjacent column.

This study received financial support from Institut Pasteur, Inserm and Santé Publique France.

\section{Cutaneous listeriosis study group}

Dr J-W Decousser (Service de Microbiologie - CHU Henri Mondor, Créteil), Dr Fazia OuldHocine (Service de Microbiologie - Hôpital Tenon, Paris), Dr E. Marsaudon (Service de médecine interne - CH de Ploermel), Dr M. Grare (Service de Microbiologie-CHU Toulouse), Pr P. Le Noc (Service de Microbiologie - CHU Grenoble), Pr H. Monteil (Service de Microbiologie - CHU Strasbourg), Dr A. Chabaud (Service de Microbiologie - CHU Strasbourg), Pr P. Pr P. Vande Perre (Service de Microbiologie - CHU Montpellier), Dr A. Smets (Laboratoire CH Draguignan), Pr A. Lozniewski (Laboratoire de Microbiologie - CHU Nancy), Pr E. Oswald (Laboratoire de Bactériologie Hygiène - Institut Fédératif de Biologie CHU Purpan, Toulouse), Dr A. Gascon (Laboratoire de Biologie, Bio Paris Ouest - LevalloisPerret), Dr C. Fabbro (Laboratoire de Biologie - Hôpital Bel-Air, Thionville), Pr B. Pozzetto (Laboratoire de Microbiologie - CHRU hôpital Nord, Saint-Etienne). 


\section{References}

1 Scobie Antonia, Kanagarajah Sanch, Harris Ross J., Byrne Lisa, Amar Corinne, Grant Kathie, et al. Mortality risk factors for listeriosis - A 10 year review of non-pregnancy associated cases in England 2006-2015. J Infect 2019;78(3):20814. Doi: 10.1016/j.jinf.2018.11.007.

2 Charlier Caroline, Leclercq Alexandre, Cazenave Benoît, Desplaces Nicole, Travier Laetitia, Cantinelli Thomas, et al. Listeria monocytogenes-associated joint and bone infections: a study of 43 consecutive cases. Clin Infect Dis Off Publ Infect Dis Soc Am 2012;54(2):240-8. Doi: 10.1093/cid/cir803.

3 McLauchlin J., Low J. C. Primary cutaneous listeriosis in adults: an occupational disease of veterinarians and farmers. Vet Rec 1994;135(26):615-7.

4 Godshall Casey E., Suh Gina, Lorber Bennett. Cutaneous listeriosis. J Clin Microbiol 2013;51(11):3591-6. Doi: 10.1128/JCM.01974-13.

5 de Valk H., Tourdjman M., Leclercq A., Maury M., Laurent E., Chenal-Francisque V., Goulet V., Brisse S. and Lecuit M. Changes in epidemiology and surveillancxe of listeriosis in France. International Symposium On Problems of Listeriosis. 2016.

6 Thouvenot Pierre, Vales Guillaume, Bracq-Dieye Hélène, Tessaud-Rita Nathalie, Maury Mylène M., Moura Alexandra, et al. MALDI-TOF mass spectrometry-based identification of Listeria species in surveillance: A prospective study. J Microbiol Methods 2018;144:29-32. Doi: 10.1016/j.mimet.2017.10.009.

7 Moura Alexandra, Tourdjman Mathieu, Leclercq Alexandre, Hamelin Estelle, Laurent Edith, Fredriksen Nathalie, et al. Real-Time Whole-Genome Sequencing for Surveillance of Listeria monocytogenes, France. Emerg Infect Dis 2017;23(9):1462-70. Doi: 10.3201/eid2309.170336.

8 Moura Alexandra, Criscuolo Alexis, Pouseele Hannes, Maury Mylène M., Leclercq Alexandre, Tarr Cheryl, et al. Whole genome-based population biology and epidemiological surveillance of Listeria monocytogenes. Nat Microbiol 2016;2:16185. Doi: 10.1038/nmicrobiol.2016.185.

9 Maury Mylène M., Tsai Yu-Huan, Charlier Caroline, Touchon Marie, Chenal-Francisque Viviane, Leclercq Alexandre, et al. Uncovering Listeria monocytogenes hypervirulence by harnessing its biodiversity. Nat Genet 2016;48(3):308-13. Doi: 10.1038/ng.3501.

10 Charlier Caroline, Perrodeau Élodie, Leclercq Alexandre, Cazenave Benoît, Pilmis Benoît, Henry Benoît, et al. Clinical features and prognostic factors of listeriosis: the MONALISA national prospective cohort study. Lancet Infect Dis 2017;17(5):510-9. Doi: 10.1016/S1473-3099(16)30521-7.

11 Bourry André, Poutrel Bernard and Rocourt Jocelyne. Bovine Mastitis caused by Listeria monocytogenes: characteristics of natural and experimental infections. J Med Microbiol 1989;43(2):125-32. 